\title{
Weaknesses and Improvements of Yang-Chang-Hwang's Password Authentication Scheme
}

\author{
Wei-Chi KU, Hao-Chuan TSAI \\ Department of Computer Science and Information Engineering, Fu Jen Catholic University \\ 510 Chung Cheng Road, Hsinchuang, Taipei County, Taiwan 242, R.O.C. \\ e-mail:wcku@csie.fju.edu.tw
}

Received: May 2004

\begin{abstract}
In 2001, Tseng, Jan, and Chien proposed an improved version of Peyravian-Zunic's password authentication scheme based on the Diffie-Hellman scheme. Later, Yang, Chang, and Hwang demonstrated that Tseng-Jan-Chien's scheme is vulnerable to a modification attack, and then described an improved scheme. In this paper, we show that Yang-Chang-Hwang's scheme is still vulnerable to a denial-of-service attack and a stolen-verifier attack. In addition, we also propose an improved scheme with better security.
\end{abstract}

Key words: password authentication, denial-of-service attack, stolen-verifier attack.

\section{Introduction}

Password authentication is a popular approach often used to authenticate users logining any kind of servers due to its simplicity and convenience. Password authentication involves the requesting entity, the user, providing a secret to the receiving entity, the server, which then checks the secret against a value stored earlier to confirm the authenticity of the user. To avoid incurring heavy computational overhead to the application system, many password authentication schemes mainly using one-way hash functions as their basic building blocks have been proposed. In 1981, Lamport (Lamport, 1981) initially proposed a password authentication scheme based on one-way hash functions. However, high hash overhead and the necessity for password resetting decrease its suitability for practical use. Additionally, Lamport's scheme is vulnerable to a preplay attack (Mitchell and Chen, 1996). In 1990, Shimizu (Shimizu, 1990) proposed a hash-based password authentication scheme, CINON, in which the one-time characteristic is gained by using two variable random numbers that are changed at each authentication. However, the user has to memorize two variable random numbers. This inconvenience obstructs the deployment of CINON. To eliminate the drawback of CINON, Shimizu, Horioka, and Inagaki (Shimizu et al., 1998) proposed an enhanced scheme, PERM, in which the user doesn't need to memorize any random number. Instead, a random number is stored in the server 
for authenticating the user. It is only when the server receives the correct response corresponding to the sent challenge, i.e., the stored random number, he will believe that the user is authentic and then refresh the stored random number. Unfortunately, PERM was found to be vulnerable to a man-in-the-middle attack (Sandirigama et al., 2000) in that the adversary can impersonate the user by modifying two consecutive sessions between the user and the server.

In 2000, Sandirigama, Shimizu, and Noda (Sandirigama et al., 2000) proposed a simple hash-based password authentication scheme, SAS, which was claimed to be superior to previous similar schemes in utilization, processing time, and transmission overhead. However, SAS was later found to be vulnerable to a replay attack, a denial-of-service attack (Lin et al., 2001), and a stolen-verifier attack (Chen and Ku, 2002). To overcome the weaknesses of SAS, Lin, Sun, and Hwang (Lin et al., 2001) proposed an improved version, OSPA. However, OSPA was found to be vulnerable to a stolen-verifier attack (Chen and $\mathrm{Ku}, 2002$ ) and a man-in-the-middle attack (Tsuji and Shimizu, 2003). Next, Lin, Shen, and Hwang (Lin et al., 2003) proposed an improved version of OSPA by additionally using smart cards. Unfortunately, Ku, Tsai, and Chen (Ku et al., 2003c) found that Lin-Shen-Hwang's scheme is still vulnerable to a denial-of-service attack and a replay attack.

Independent of the above mentioned schemes, Peyravian and Zunic (Peyravian and Zunic, 2000) also proposed a hash-based password authentication scheme, which involves the protected password transmission protocol and the protected password change protocol. Later, Hwang and Yeh (Hwang and Yeh, 2002) showed that Peyravian-Zunic's scheme is vulnerable to an off-line password guessing attack, a server spoofing attack, and a server data eavesdropping attack, and then proposed a modified scheme. However, Hwang-Yeh's scheme is inefficient and inconvenient because of using the publickey cryptosystem. Furthermore, Hwang-Yeh's scheme was found to be vulnerable to a denial-of-service attack and the threat of a replay attack (Ku et al., 2003a). Next, Lee, $\mathrm{Li}$, and Hwang (Lee et al., 2002) proposed another improvement of Peyravian-Zunic's scheme. However, $\mathrm{Ku}$, Chen, and Lee (Ku et al., 2003b) demonstrated that Lee-LiHwang's scheme is still vulnerable to a denial-of-service attack, a stolen-verifier attack, and an off-line password guessing attack. In 2001, Tseng, Jan, and Chien (Tseng et al., 2001) also pointed out the weaknesses of Peyravian-Zunic's scheme, and then proposed an improved version based on the Diffie-Hellman scheme (Diffie and Hellman, 1976). However, Tseng-Jan-Chien's scheme was found to be vulnerable to a modification attack (Yang et al., 2003), which is a kind of denial-of-service attacks, and a stolen-verifier attack (Hsieh et al., 2003). Recently, Yang, Chang, and Hwang (Yang et al., 2003) proposed an improved version of Tseng-Jan-Chien's scheme and claimed that their scheme is secure against the password guessing attack, the replay attack, the server spoofing attack, and the modification attack. Unfortunately, we find that Yang-Chang-Hwang's scheme is still vulnerable to a denial-of-service attack and a stolen-verifier attack. In this paper, we will first review Yang-Chang-Hwang's scheme, and then describe its weaknesses. The rest of this paper is organized as follows. In Section 2, we briefly review Yang-ChangHwang's scheme. Then, we show the weaknesses of Yang-Chang-Hwang's scheme in 
Section 3. Then, we will propose an improved scheme with better resistances to the denial-of-service attack and the stolen-verifier attack in Section 4. Finally, conclusions are made in Section 5.

\section{Review of Yang-Chang-Hwang's Scheme}

Yang-Chang-Hwang's scheme (Yang et al., 2003) involves the protected password transmission protocol and the protected password change protocol. Its protected password transmission protocol is identical to the one of Tseng-Jan-Chien's scheme while its protected password change protocol was intended to be an improvement of the one of TsengJan-Chien's scheme. For readers' convenience, we will review Yang-Chang-Hwang's scheme before demonstrating its weaknesses. The notations used throughout this paper are described as follows. Notations $i d$ and $p w$ represent the identity and the password of the client, respectively. $H(\cdot)$ denotes a one-way hash function and $\oplus$ represents the bitwise XOR operation. Initially, the server publishes two large prime numbers $p$ and $q$ such that $q \mid p-1$ and a generator $g$ with order $q$ in the Galois field $G F(p)$. To register as a user, the client computes $h p w=H(i d, p w)$, and delivers $h p w$ along with $i d$ to the server through a secure channel. Then, the server creates an entry in the database for the client to store $h p w$ as the verifier of $p w$. The protected password transmission protocol and the protected password change protocol of Yang-Chang-Hwang's scheme can be described as in the following.

\subsection{Protected Password Transmission Protocol}

The protected password transmission protocol is invoked whenever the client requests to login the server by using $p w$.

Step T1. Client $\longrightarrow$ Server: $i d, h p w \oplus r c$.

The client enters $i d$ and $p w$ to compute $h p w=H(i d, p w)$, and chooses a random number $c \in[1, q-1]$ to compute $r c=g^{c} \bmod p$ and $h p w \oplus r c$. Next, the client sends $i d$ and $h p w \oplus r c$ to the server.

Step T2. Server $\longrightarrow$ Client: $h p w \oplus r s, H(h p w, r c s, r c)$.

The server retrieves $r c$ from the second item of the message received in Step T1 by using the stored verifier $h p w$. In addition, the server chooses a random number $s \in$ $[1, q-1]$ to compute $r s=g^{s} \bmod p$ and $h p w \oplus r s$. Next, the server computes $r c s=$ $r c^{s}=g^{c s} \bmod p$, and then uses $h p w$, the computed $r c s$, and the retrieved $r c$ to compute $H(h p w, r c s, r c)$. Then, the server sends $h p w \oplus r s$ and $H(h p w, r c s, r c)$ to the client.

Step T3. Client $\longrightarrow$ Server: $i d, H(h p w, r c s, r s)$.

The client retrieves $r s$ from the first item of the message received in Step T2 by using $h p w$, and then computes $r c s=r s^{c}=g^{s c} \bmod p$. Next, the client uses $h p w, r c$, and $r c s$ to compute $H(h p w, r c s, r c)$. If the computed result equals the second item of the 
message received in Step T2, the server is authenticated. Next, the client uses hpw, rcs, and $r s$ to compute $H(h p w, r c s, r s)$, and then sends $i d$ and $H(h p w, r c s, r s)$ to the server.

\section{Step T4. Server $\longrightarrow$ Client: Access Granted/Denied.}

The server uses $r c s, r s$, and the stored verifier $h p w$ to compute $H(h p w, r c s, r s)$. If the computed result equals the second item of the message received in Step T3, the server grants the client's login request and sends 'Access Granted' to the client. Otherwise, the server rejects the client's login request and sends 'Access Denied' to the client. After successful mutual authentication, the client and the server can compute $H(r c s)$ and use it as the session key for protecting the messages exchanged between them in this session.

\subsection{Protected Password Change Protocol}

The protected password change protocol is invoked whenever the client requests to change his password $p w$ with a new one, say $p w_{n e w}$.

Step C1. Client $\longrightarrow$ Server: $i d, h p w \oplus r c, h p w_{n e w} \oplus r c$.

The client enters $i d$ and $p w$ to compute $h p w=H(i d, p w)$ and chooses a random number $c \in[1, q-1]$ to compute $r c=g^{c} \bmod p$ and $h p w \oplus r c$. In addition, the client chooses his new password $p w_{n e w}$, and then computes $h p w_{n e w}=H\left(i d, p w_{n e w}\right)$ and $h p w_{\text {new }} \oplus r c$. Next, the client sends $i d, h p w \oplus r c$, and $h p w_{n e w} \oplus r c$ to the server.

Step C2. Server $\longrightarrow$ Client: $h p w \oplus r s, H(h p w, r c s, r c)$.

The server retrieves $r c$ from the second item of the message received in Step C1 by using the stored verifier $h p w$, and then uses the retrieved $r c$ to retrieve $h p w_{n e w}$ from the third item of the message received in Step C1. Next, the server chooses a random number $s \in[1, q-1]$ to compute $r s=g^{s} \bmod p$ and $h p w \oplus r s$. In addition, the server computes $r c s=r c^{s}=g^{c s} \bmod p$, and then uses the stored verifier $h p w$, the computed $r c s$, and the retrieved $r c$ to compute $H(h p w, r c s, r c)$. Next, the server sends $h p w \oplus r s$ and $H(h p w, r c s, r c)$ to the client.

Step C3. Client $\longrightarrow$ Server: $i d, H(h p w, r c s, r s) \oplus h p w_{n e w}$.

The client retrieves rs from the first item of the message received in Step C2 by using $h p w$, and then computes $r c s=r s^{c}=g^{s c} \bmod p$. Next, the client uses hpw, rcs, and $r c$ to compute $H(h p w, r c s, r c)$. If the computed result equals the second item of the message received in Step C2, the server is authenticated. After successfully authenticating the server, the client uses $r c s, h p w, h p w_{n e w}$, and the retrieved $r s$ to compute $H(h p w, r c s, r s) \oplus h p w_{n e w}$, and then sends $i d$ and $H(h p w, r c s, r s) \oplus h p w_{n e w}$ to the server.

\section{Step C4. Server $\longrightarrow$ Client: Accepted/Denied.}

The server uses the previously obtained $h p w_{n e w}$ to retrieve $H(h p w, r c s, r s)$ from the second item of the message received in Step C3, and uses the stored verifier $h p w$, the previously computed $r c s$, and $r s$ to compute $H(h p w, r c s, r s)$. If the computed 
$H(h p w, r c s, r s)$ equals the retrieved one, the server authenticates the client, and then updates the stored verifier $h p w$ with $h p w_{n e w}$ and sends 'Accepted' to the client. Otherwise, the server sends 'Denied' to the client.

\section{Weaknesses of Yang-Chang-Hwang's Scheme}

In this section, we will show that Yang-Chang-Hwang's scheme (Yang et al., 2003) is vulnerable to a denial-of-service attack and a stolen-verifier attack.

\subsection{Denial-of-Service Attack}

The denial-of-service attack is an attack leading a legal user can not login the server or the server can not provide service normally. Next, we will demonstrate the way to mount a denial-of-service attack on Yang-Chang-Hwang's scheme. Suppose that the client requests to change his password $p w$ with a new one, say $p w_{n e w}$, by invoking the protected password change protocol. During Step C1, an adversary, say Eve, can replace the transmitting $h p w_{\text {new }} \oplus r c$ with $\left(h p w_{\text {new }} \oplus r c\right) \oplus r e$, where $r e$ is a random number selected by Eve. Upon receiving the modified message in Step C1, the server will retrieve $r c$ by using the stored verifier $h p w$, and then use the retrieved $r c$ to retrieve $h p w_{\text {new }} \oplus r e$ from the received $\left(h p w_{\text {new }} \oplus r c\right) \oplus r e\left(=\left(h p w_{\text {new }} \oplus r e\right)\right.$ $\oplus r c)$. Next, the server will choose a random number $s \in[1, q-1]$ to compute $r s=g^{s} \bmod p, h p w \oplus r s, r c s=r c^{s}=g^{c s} \bmod p$, and $H(h p w, r c s, r c)$. Then, the server sends $h p w \oplus r s$ and $H(h p w, r c s, r c)$ to the client in Step C2. Upon receiving the message received in Step C2, the client will successfully authenticate the server. Then, the client will send $i d$ and $H(h p w, r c s, r s) \oplus h p w_{n e w}$ to the server in Step C3. During Step C3, Eve can replace the transmitting $H(h p w, r c s, r s) \oplus h p w_{n e w}$ with $\left(H(h p w, r c s, r s) \oplus h p w_{n e w}\right) \oplus r e$. Upon receiving the modified message in Step C3, the server will use the previously obtained $h p w_{\text {new }} \oplus r e$ to retrieve $H(h p w, r c s, r s)$ from the received $\left(H(h p w, r c s, r s) \oplus h p w_{\text {new }}\right) \oplus r e\left(=\left(h p w_{\text {new }} \oplus r e\right) \oplus H(h p w, r c s, r s)\right)$. In addition, the server will use $h p w, r c s$, and $r s$ to compute $H(h p w, r c s, r s)$. Since the computed $H(h p w, r c s, r s)$ equals the retrieved one, the server authenticates the client and then changes the stored verifier $h p w$ with $h p w_{\text {new }} \oplus r e$, which clearly does not equal the expected $h p w_{\text {new }}$. That is, the server is fooled into taking $h p w_{\text {new }} \oplus r e$ as the client's new verifier. Henceforth, the client's login and password change requests using $h p w_{\text {new }}$ will be denied. Since the adversary can easily block the account of any client without using cryptographic techniques, Yang-Chang-Hwang's scheme is vulnerable to a denial-of-service attack.

\subsection{Stolen-Verifier Attack}

In most existing password authentication schemes, the server stores the verifiers of users' passwords rather than users' bare passwords to reduce the risk once the server is compromised. The stolen-verifier attack is an offensive attack that the adversary who has 
stolen the user's verifier can impersonate that user and/or perform other attacks. Next, we will describe the way to mount a stolen-verifier attack on Yang-Chang-Hwang's scheme. Clearly, once an adversary, say Eve, has stolen an ever used verifier, she can compute its succeeding verifier if she has also intercepted the corresponding message transmitted in Step C1. By iteratively applying the above method, Eve can obtain the client's current verifier $h p w$. Suppose that Eve has obtained, either directly or indirectly, the client's current verifier $h p w$. First, Eve can choose a random number $e$ to compute $r e=g^{e} \bmod p$ and $h p w \oplus r e$, and then impersonate the client to send $i d$ and $h p w \oplus r e$ to the server in Step T1 of the protected password transmission protocol. Upon receiving the forged message, the server will use the stored verifier $h p w$ to retrieve re from the second received item, and then choose a random number $s$ to compute $r s=g^{s} \bmod p, h p w \oplus r s$, $r e s=r e^{s}=g^{e s} \bmod p$, and $H(h p w$,res, re $)$. Next, the sever will send hpw $\oplus$ rs and $H(h p w, r e s, r e)$ to Eve. Then, Eve can use the stolen verifier $h p w$ to retrieve $r s$ from the first received item, and then compute $r e s=r s^{e}=g^{s e} \bmod p$ and $H$ (hpw, res, rs). Next, Eve sends $i d$ and $H(h p w, r e s, r s)$ to the server in Step T3. Then, the server will use the stored verifier $h p w$ and the previously computed res and $r s$ to compute $H(h p w, r e s, r s)$. Since the computed $H$ ( hpw, res, rs) equals the second item of the received message, the server will grant Eve's login request, i.e., Eve can successfully impersonate the client to login the server and obtain the resources or services she needs. Similarly, Eve can also use the stolen verifier $h p w$ to change the client's password $p w$ with her own password $p w_{e}$ by invoking the protected password change protocol. As the client's verifier will be replaced with $h p w_{e}\left(=H\left(i d, p w_{e}\right)\right)$, Eve can use $p w_{e}$ to impersonate the client to login the server. In this case, the client's succeeding login requests using $p w$ will be denied.

Alternatively, knowing $h p w$, Eve can also perform a man-in-the-middle attack as follows. During the client's login process, Eve can use the stolen hpw to impersonate the server to fool the client into establishing a session key with her and simultaneously impersonate the client to fool the server into establishing another session key with her. Therefore, Eve can decrypt all the encrypted messages exchanged between the client and the server. Clearly, such an attack can not be easily detected.

\section{An Improved Version of Yang-Chang-Hwang's Scheme}

In this section, we will describe an improved version of Yang-Chang-Hwang's scheme with better security strength.

\subsection{The Improved Scheme}

As in Yang-Chang-Hwang's scheme, the server initially publishes two large prime numbers $p$ and $q$ such that $q \mid p-1$ and a generator $g$ with order $q$ in $G F(p)$. To register as a user, the client computes $h p w=H(i d, p w)$ and delivers $h p w$ along with $i d$ to the server through a secure channel. Then, the server computes enc_hpw $=h p w \oplus H(x, i d)$, where $x$ is a secret storage key of the server, and creates an entry in the database to store 
enc_hpw for the client. It is assumed that $x$ is under strict protection. The improved protected password transmission protocol and protected password change protocol can be described as in the following.

\subsubsection{Improved Protected Password Transmission Protocol}

The improved protected password transmission protocol is identical to the one of YangChang-Hwang's scheme except that Step T2 is slightly changed into Step T2' as follows:

Step $\mathrm{T}^{\prime}$. Server $\longrightarrow$ Client: $h p w \oplus r s, H(h p w, r c s, r c)$.

The server computes $H(x, i d)$ and then uses the result to retrieve $h p w$ from the stored enc.hpw $(=h p w \oplus H(x, i d))$. Next, the server uses the retrieved $h p w$ to retrieve $r c$ from the second item of the message received in Step T1. In addition, the server chooses a random number $s \in[1, q-1]$ to compute $r s=g^{s} \bmod p$ and $h p w \oplus r s$. Next, the server computes $r c s=r c^{s}=g^{c s} \bmod p$, and then uses $h p w, r c s$, and $r c$ to compute $H(h p w, r c s, r c)$. Then, the server sends $h p w \oplus r s$ and $H(h p w, r c s, r c)$ to the client. As in Yang-Chang-Hwang's scheme, the client and the server can compute $H(r c s)$ after successful mutual authentication and then use it as the session key for protecting the messages exchanged between them in this session.

\subsubsection{Improved Protected Password Change Protocol}

Steps $\mathrm{C} 1, \mathrm{C} 2, \mathrm{C} 3$, and $\mathrm{C} 4$ of Yang-Chang-Hwang's scheme are changed into $\mathrm{C1}^{\prime}, \mathrm{C}^{\prime}$, $\mathrm{C}^{\prime}$, and $\mathrm{C}^{\prime}$, respectively, as follows:

Step $\mathrm{C1}^{\prime}$. Client $\longrightarrow$ Server: $i d, h p w \oplus r c$.

The client enters $i d$ and $p w$ to compute $h p w=H(i d, p w)$ and chooses a random number $c \in[1, q-1]$ to compute $r c=g^{c} \bmod p$ and $h p w \oplus r c$. Next, the client sends $i d$ and $h p w \oplus r c$ to the server.

Step $\mathrm{C} 2^{\prime}$. Server $\longrightarrow$ Client: $h p w \oplus r s, H(h p w, r c s, r c)$.

The server computes $H(x, i d)$ and then uses the result to retrieve $h p w$ from the stored enc_hpw $(=h p w \oplus H(x, i d))$. Next, the server uses the retrieved $h p w$ to retrieve $r c$ from the second item of the message received in Step $\mathrm{C}^{\prime}{ }^{\prime}$. In addition, the server chooses a random number $s \in[1, q-1]$ to compute $r s=g^{s} \bmod p$ and $h p w \oplus r s$. Next, the server computes $r c s=r c^{s}=g^{c s} \bmod p$, and then uses $h p w, r c s$, and $r c$ to compute $H(h p w, r c s, r c)$. Then, the server sends $h p w \oplus r s$ and $H(h p w, r c s, r c)$ to the client.

Step $\mathrm{C} 3^{\prime}$. Client $\longrightarrow$ Server: $i d, H(h p w, r c s, r s) \oplus h p w_{n e w}, H\left(r c s, h p w_{n e w}\right)$.

The client retrieves $r s$ from the first item of the message received in Step $\mathrm{C}^{\prime}$ by using $h p w$, and then computes $r c s=r s^{c}=g^{s c} \bmod p$. Next, the client uses hpw, $r c s$, and $r c$ to compute $H(h p w, r c s, r c)$. If the computed result equals the second item of the message received in Step $\mathrm{C}^{\prime}$, the server is authenticated. Then, the client chooses his new password $p w_{n e w}$, and then computes $h p w_{\text {new }}=H\left(i d, p w_{\text {new }}\right)$. Next, the client uses $r c s, h p w, h p w_{\text {new }}$, and the retrieved $r s$ to compute $H(h p w, r c s, r s) \oplus h p w_{\text {new }}$ and 
$H\left(r c s, h p w_{n e w}\right)$, and then sends $i d, H(h p w, r c s, r s) \oplus h p w_{n e w}$, and $H\left(r c s, h p w_{n e w}\right)$ to the server.

Step $\mathrm{C} 4^{\prime}$. Server $\longrightarrow$ Client: Accepted/Denied.

The server uses hpw, rcs, and $r s$ to compute $H(h p w, r c s, r s)$ and then uses the computed result to retrieve $h p w_{n e w}$ from the second item of the message received in Step C3'. Next, the server uses $r c s$ and the retrieved $h p w_{n e w}$ to compute $H\left(r c s, h p w_{n e w}\right)$. If the computed result equals the third item of the message received in Step C3', the server authenticates the client, and then updates the stored enc_hpw with enc_hpw $w_{n e w}=$ $h p w_{\text {new }} \oplus H(x, i d)$ and sends 'Accepted' to the client. Otherwise, the server rejects the client's login request and sends 'Denied' to the client.

\subsection{Security Analysis of The Improved Scheme}

The resistances of the improved scheme to the replay attack, the password guessing attack, and the server spoofing attack are similar to the ones of Yang-Chang-Hwang's scheme (Yang et al., 2003), and we omit the proofs for clearness. Next, we will show that the improved scheme can additionally resist the denial-of-service attack and the stolenverifier attack. Let Eve denote the adversary.

\subsubsection{Resistance to Denial-of-Service Attack}

Suppose that the client requests to change his password $p w$ with $p w_{n e w}$ by invoking the improved protected password change protocol. Upon receiving the client's request sent in Step $\mathrm{C}^{\prime}$, the server will compute $H(x, i d)$ to retrieve $h p w$ from the stored enc_hpw. Next, the server will use $h p w$ to retrieves $r c$ from the received $h p w \oplus r c$. In addition, the server will choose a random number $s \in[1, q-1]$ to compute $r s=g^{s} \bmod p, h p w \oplus r s, r c s=r c^{s}=g^{c s} \bmod p$, and $H(h p w, r c s, r c)$, and then send $h p w \oplus r s$ and $H(h p w, r c s, r c)$ to the client in Step $\mathrm{C}^{\prime}$. Upon receiving the message sent in Step $\mathrm{C}^{\prime}$, the client will successfully authenticate the server and send $i d$, $H(h p w, r c s, r s) \oplus h p w_{n e w}$, and $H\left(r c s, h p w_{n e w}\right)$ to the server in Step C3'. If Eve can replace the transmitting $H(h p w, r c s, r s) \oplus h p w_{n e w}$ and $H\left(r c s, h p w_{n e w}\right)$ with $\left(H(h p w, r c s, r s) \oplus h p w_{n e w}\right) \oplus r e$ and $H\left(r c s, h p w_{n e w} \oplus r e\right)$, where $r e$ is a random number selected by Eve, respectively, she can fool the server into updating the stored $e_{n} c_{-} h p w$ with $h p w_{n e w} \oplus r e \oplus H(x, i d)$ so that the client's subsequent login requests and password change requests will be denied. However, Eve can not produce $H\left(r c s, h p w_{n e w} \oplus r e\right)$ because she can not obtain $r c s$ and $h p w_{n e w}$ by analyzing the protocol messages. Thus, the improved scheme can resist the denial-of-service attack.

\subsubsection{Resistance to Stolen-Verifier Attack}

Suppose that Eve has stolen enc_hpw. As enc_hpw $=h p w \oplus H(x, i d)$, Eve can derive $h p w$ only if she knows $H(x, i d)$, which implies that she knows $x$, the secret storage key of the server. However, since $x$ is under strict protection as assumed, it is infeasible for Eve to derive $h p w$ in this way. Therefore, the improved scheme can prevent from the stolen-verifier attack. 


\section{Conclusion}

Herein, we have shown that Yang-Chang-Hwang's password authentication scheme, which was intended to be an improved version of Tseng-Jan-Chien's password authentication scheme, is still vulnerable to a denial-of-service attack and a stolen-verifier attack. As analyzed, the security flaws of Yang-Chang-Hwang's scheme are due to two problems. First, the integrity of the transmitted messages from the user to the server in the protected password change protocol are not well protected. The adversary can modify the transmitted messages to block the account of the user without being detected by the server. Secondly, although the server stores the verifier of the user's password rather than the user's bare password to reduce the risk once the server is compromised, the adversary can still use the stolen verifier to impersonate the user. Furthermore, we have described an improved version of Yang-Chang-Hwang's scheme with better resistances to the denialof-service attack and the stolen-verifier attack.

\section{Acknowledgement}

We are grateful to the referees for their valuable comments. This research was supported by the National Science Council, Republic of China, under Grant NSC-92-2213-E-030013.

\section{References}

Chen, C.M., and W.C. Ku (2002). Stolen-verifier attack on two new strong-password authentication protocols IEICE Transactions on Communications, E85-B(11), 2519-2521.

Diffie, W., and M. Hellman (1976). New direction in cryptography. IEEE Transactions on Information Theory, 22(6), 472-492.

Hsieh, B.T., H.M. Sun and T. Hwang (2003). On the security of some password authentication protocols. Informatica, 14(2), 195-204.

Hwang, J.J., and T.C. Yeh (2002). Improvement on Peyravian-Zunic's password authentication schemes. IEICE Transactions on Communications, E85-B(4), 823-825.

Ku, W.C., C.M. Chen and H.L. Lee (2003a). Cryptanalysis of a variant of Peyravian-Zunic's password authentication scheme. IEICE Transactions on Communications, E86-B(5), 1682-1684.

$\mathrm{Ku}$, W.C., C.M. Chen and H.L. Lee (2003b). Weaknesses of Lee-Li-Hwang's hash-based password authentication scheme. ACM Operating Systems Review, 37(4), 19-25.

Ku, W.C., H.C. Tsai and S.M. Chen (2003c). Two simple attacks on Lin-Shen-Hwang's strong-password authentication protocol. ACM Operating Systems Review, 37(4), 26-31.

Lamport, L. (1981). Password authentication with insecure communication. Communications of the ACM, 24(11), 770-772

Lee, C.C., L.H. Li and M.S. Hwang (2002). A remote user authentication scheme using hash functions. ACM Operating Systems Review, 36(4), 23-29.

Lin, C.L., H.M. Sun and T. Hwang (2001). Attacks and solutions on strong-password authentication. IEICE Transactions on Communications, E84-B(9), 2622-2627.

Lin, C.W., J.J. Shen and M.S. Hwang (2003). Security enhancement for optimal strong password authentication protocol. ACM Operating Systems Review, 37(2), 7-12.

Mitchell, C.J., and L. Chen (1996). Comments on the S/KEY user authentication scheme. ACM Operating Systems Review, 30(4), 12-16. 
Peyravian, M., and N. Zunic (2000). Methods for protecting password transmission. Computers and Security, 19(5), 466-469.

Sandirigama, M., A. Shimizu and M.T. Noda (2000). Simple and secure password authentication protocol (SAS). IEICE Transactions on Communications, E83-B(6), 1363-1365.

Shimizu, A. (1990). A dynamic password authentication method by one-way function. IEICE Transactions, J73-D-I(7), 630-636.

Shimizu, A., T. Horioka and H. Inagaki (1998). A password authentication methods for contents communication on the Internet. IEICE Transactions on Communications, E81-B(8), 1666-1673.

Tseng, Y.M., J.K. Jan and H.Y. Chien (2001). On the security of methods for protecting password transmission. Informatica, 12(3), 469-477.

Tsuji, T., and A. Shimizu (2003). An impersonation attack on one-time password authentication protocol OSPA. IEICE Transactions on Communications, E86-B(7), 2182-2185.

Yang, C.C., T.Y. Chang and M.S. Hwang (2003). Security of improvement on methods for protecting password transmission. Informatica, 14(4), 551-558.

W.C. Ku was born in Taiwan in 1967. In 2000, he received the PhD degree in Electrical Engineering from National Taiwan University. In 2001, Dr. Ku joined the faculty of the Department of Computer Science and Information Engineering at Fu Jen Catholic University, where he is currently an associate professor. His research interests include cryptography and information security.

H.C. Tsai was born in Taiwan in 1978. In 2002, he received the BS degree in Mathematics from SooChow University, Taiwan. He is currently a master's student in computer science and information engineering at Fu Jen Catholic University. His research interests include cryptography and information security.

\section{Yang-Chang-Hwang slaptažodžio autentiškumo schemos trūkumai ir pagerinimai}

\section{Wei-Chi KU, Hao-Chuan TSAI}

Tseng, Jan ir Chien 2001 buvo pasiūlyta pagerinta Peyravian-Zunic slaptažodžio autentiškumo schema, pagrịsta Diffie-Hellman schema. Vèliau, Yang, Chang ir Hwang pademonstravo, kad Tseng-Jan-Chien schema yra pažeidžiama modifikavimo ataka, ir pasiūlè pagerintą schemą. Šiame straipsnyje parodoma, kad Yang-Chang-Hwang schema yra pažeidžiama serviso-paneigimo ir pavogto-tikrintojo atakų. Dèl to geresniam saugumui pasiūlyta pagerinta schema. 